\title{
EXISTENCE RESULTS FOR MINIMIZERS OF PARAMETRIC ELLIPTIC FUNCTIONALS
}

\author{
GUIDO DE PHILIPPIS, ANTONIO DE ROSA, AND FRANCESCO GHIRALDIN
}

\begin{abstract}
We prove a compactness principle for the anisotropic formulation of the Plateau problem in any codimension, in the same spirit of the previous works of the authors DGM14, DPDRG15, DLDRG16]. In particular, we perform a new strategy for the proof of the rectifiability of the minimal set, based on the new anisotropic counterpart of the Allard rectifiability theorem proved in DPDRG16]. As a consequence we provide a new proof of the Reifenberg existence theorem.
\end{abstract}

\section{Introduction and Main Result}

This paper concludes a series of works by the authors on the Plateau problem: we here provide a general and flexible existence result for sets that minimize an anisotropic energy, which can be applied to several notions of boundary conditions.

In the spirit of the previous works [DGM14], [DPDRG15] and [DLDRG16], we use the direct method of the calculus of variations to find a generalised minimizer (namely a Radon measure) via standard compactness arguments, and then we aim at proving that it is actually a fairly regular surface. To do this, we employed several techniques to first establish the rectifiability of the limit measure: in the case of the area integrand this property was initially deduced from a powerful result due to Preiss [Pre87, De 08], as well as, in codimension one, from the theory of sets of finite perimeter. These two techniques are no longer available in the case of anisotropic problems in higher codimension (in particular due to the lack of a monotonicity formula for anisotropic problems). A new rectifiability criterion was found in [DPDRG16, Theorem 1.2], for varifolds having positive lower density and a bounded anisotropic first variation, extending the celebrated result by Allard [All72], see also De 16].

The proof of the existence theorem can be applied to the minimization of the energy in several classes of sets, corresponding to several notions of boundary conditions as the one used in [HP15, HP16]. In particular we discuss the existence theorem for minimizers with a homological notion of boundary, originally considered by Reifenberg in the isotropic case [Rei60], see Section 3, Our techniques can as well be extended to prove existence for the anisotropic Plateau problem under co-homological boundary conditions, first considered in [HP16] where however more general assumptions on the integrand are allowed, see Remark 1.8 and 3.5 below. Recently two related existence theorems have been proved in [Fan16] and in [FK17], following the strategy of [Alm68, Alm76]. In particular in [Fan16] an existence Theorem in the Reifenberg setting is extended to the case of $\mathbb{Z}$ coefficients, see the discussion after Theorem 3.4 below.

In order to precisely state our main result, we introduce some notations and definitions. We will always work in $\mathbb{R}^{n}$ and $1 \leq d \leq n$ will always be an integer number. We recall that a set $K$ is said to be $d$-rectifiable if it can be covered, up to an $\mathcal{H}^{d}$ negligible set, by countably many $C^{1}$ manifolds where $\mathcal{H}^{d}$ is the $d$-dimensional Hausdorff measure. We also denote by $G=G(n, d)$ the Grassmannian of unoriented $d$-dimensional linear subspaces of $\mathbb{R}^{n}$ and, for every $U \subset \mathbb{R}^{n}$, we define $G(U):=U \times G$. Given a $d$-rectifiable set $K$, we denote by $T_{K}(x)$ the approximate

2010 Mathematics Subject Classification. 49Q20 (28A75 49Q15). 
tangent space of $K$ at $x$, which exists for $\mathcal{H}^{d}$-almost every point $x \in K$ [Sim83, Chapter 3]. We also let $\operatorname{Lip}\left(\mathbb{R}^{n}\right)$ be the space of Lipschitz maps in $\mathbb{R}^{n}$.

The anisotropic Lagrangians considered in the rest of the note will be $C^{1}$ maps

$$
F: \mathbb{R}^{n} \times G \ni(x, T) \mapsto F(x, T) \in \mathbb{R}^{+},
$$

verifying the lower and upper bounds

$$
0<\lambda \leq F(x, T) \leq \Lambda<\infty .
$$

Given a $d$-rectifiable set $K$ and an open subset $U \subset \mathbb{R}^{n}$, we define:

$$
\mathbf{F}(K, U):=\int_{K \cap U} F\left(x, T_{K}(x)\right) d \mathcal{H}^{d}(x) \text { and } \mathbf{F}(K):=\mathbf{F}\left(K, \mathbb{R}^{n}\right) .
$$

It will be also useful to look at the "frozen" functional: for $y \in \mathbb{R}^{n}$, we let

$$
\mathbf{F}^{y}(K, U):=\int_{K \cap U} F\left(y, T_{K}(x)\right) d \mathcal{H}^{d}(x) .
$$

We note that given a $d$-dimensional varifold $V$ (i.e., a positive Radon measure on the Grassmannian $G(U))$ we can define its anisotropic energy as

$$
\mathbf{F}(V, U):=\int F(x, T) d V(x, T) ;
$$

this definition is coherent with (1.2), since to any rectifiable set $K$ we will naturally associate the varifold $\mathcal{H}^{d}\left\llcorner K \otimes \delta_{T_{K}(x)}\right.$. In this setting we define the anisotropic first variation of a varifold $V$ as the order one distribution whose action on $g \in C_{c}^{1}\left(U, \mathbb{R}^{n}\right)$ is given by

$$
\begin{aligned}
\delta_{F} V(g) & :=\left.\frac{d}{d t} \mathbf{F}\left(\varphi_{t}^{\#} V\right)\right|_{t=0} \\
& =\int_{\Omega \times G(n, d)}\left[\left\langle d_{x} F(x, T), g(x)\right\rangle+B_{F}(x, T): D g(x)\right] d V(x, T),
\end{aligned}
$$

where $\varphi_{t}(x)=x+\operatorname{tg}(x), \varphi_{t}^{\#} V$ is the image varifold of $V$ through $\varphi_{t}$ see Sim83, Chapter 8], $B_{F}(x, T) \in \mathbb{R}^{n} \otimes \mathbb{R}^{n}$ is an explicitly computable $n \times n$ matrix and $\langle A, B\rangle:=\operatorname{tr} A^{*} B$ for $A, B \in \mathbb{R}^{n} \otimes \mathbb{R}^{n}$, see for instance [DPDRG16] for the relevant computations. A varifold $V$ is said to be $F$-stationary in an open set $U$ if $\delta_{F} V=0$ as a distribution in $U$.

Throughout all the paper, $H \subset \mathbb{R}^{n}$ will denote a closed subset of $\mathbb{R}^{n}$. Assume to have a class $\mathcal{P}(H)$ of relatively closed $d$-rectifiable subsets $K$ of $\mathbb{R}^{n} \backslash H$. One can then formulate the anisotropic Plateau problem by asking whether the infimum

$$
m_{0}:=\inf \{\mathbf{F}(K): K \in \mathcal{P}(H)\}
$$

is achieved by some set (which should be a suitable limit of a minimizing sequence). A second question is whether this set belongs to the chosen class $\mathcal{P}(H)$ and which additional regularity properties it satisfies. We will say that a sequence $\left\{K_{j}\right\} \subset \mathcal{P}(H)$ is a minimizing sequence if $\mathbf{F}\left(K_{j}\right) \downarrow m_{0}$.

We next outline a set of flexible and rather weak requirements for $\mathcal{P}(H)$ : the key property for $K^{\prime}$ to be a competitor of $K$ is that $K^{\prime}$ is close in energy to sets obtained from $K$ via deformation maps as in Definition 1.1. This allows for a slightly larger flexibility on the choice of the admissible sets, since a priori $K^{\prime}$ might not belong to the competition class.

Definition 1.1 (Lipschitz deformations). Given a ball $B_{x, r}$, we let $\mathfrak{D}(x, r)$ be the set of functions $\varphi: \mathbb{R}^{n} \rightarrow \mathbb{R}^{n}$ such that $\varphi(z)=z$ in $\mathbb{R}^{n} \backslash B_{x, r}$ and which are smoothly isotopic to the identity inside $B_{x, r}$, namely those for which there exists an isotopy $\lambda \in C^{\infty}\left([0,1] \times \mathbb{R}^{n} ; \mathbb{R}^{n}\right)$ such that

$$
\begin{gathered}
\lambda(0, \cdot)=\mathrm{Id}, \quad \lambda(1, \cdot)=\varphi, \quad \lambda(t, y)=y \quad \forall(t, y) \in[0,1] \times\left(\mathbb{R}^{n} \backslash B_{x, r}\right) \quad \text { and } \\
\lambda(t, \cdot) \text { is a diffeomorphism of } \mathbb{R}^{n} \forall t \in[0,1] .
\end{gathered}
$$


We finally set $\mathrm{D}(x, r):=\overline{\mathfrak{D}(x, r)} w^{*}-W^{1, \infty}$, the sequential closure of $\mathfrak{D}(x, r)$ with respect to the uniform convergence with equibounded differentials.

Observe that in the definition of $\mathrm{D}(x, r)$ it is equivalent to require any $C^{k}$ regularity on the isotopy $\lambda$, for $k \geq 1$, as $C^{k}$ isotopies coinciding with the identity outside $B_{x, r}$ can be approximated in $C^{k}$ by smooth ones also supported in the same set.

Definition 1.2 (Deformed competitors and good class). Let $H \subset \mathbb{R}^{n}$ be closed, $K \subset \mathbb{R}^{n} \backslash H$ be a relatively closed countably $\mathcal{H}^{d}$-rectifiable set and $B_{x, r} \subset \mathbb{R}^{n} \backslash H$. A deformed competitor for $K$ in $B_{x, r}$ is any set of the form

$$
\varphi(K) \quad \text { where } \quad \varphi \in \mathrm{D}(x, r) .
$$

Given a family $\mathcal{P}(H)$ of relatively closed $d$-rectifiable subsets $K \subset \mathbb{R}^{n} \backslash H$, we say that $\mathcal{P}(H)$ is a good class if for every $K \in \mathcal{P}(H)$, for every $x \in K$ and for a.e. $r \in(0$, dist $(x, H))$

$$
\inf \left\{\mathbf{F}(J): J \in \mathcal{P}(H), J \backslash \overline{B_{x, r}}=K \backslash \overline{B_{x, r}}\right\} \leq \mathbf{F}(L)
$$

whenever $L$ is any deformed competitor for $K$ in $B_{x, r}$.

We will assume the following ellipticity condition on the energy $\mathbf{F}$, introduced in Alm68], which is a geometric version of quasiconvexity, cf. [Mor66]:

Definition 1.3 (Elliptic integrand, $[\mathrm{Alm68}, 1.2]$ ). The anisotropic Lagrangian $F$ is said to be elliptic if there exists $\Gamma \geq 0$ such that, whenever $x \in \mathbb{R}^{n}$ and $D$ is a $d$-disk centered in $x$, with radius $r$ and contained in a $d$-plane $T$, then the inequality

$$
\mathbf{F}^{x}\left(K, B_{x, r}\right)-\mathbf{F}^{x}\left(D, B_{x, r}\right) \geq \Gamma\left(\mathcal{H}^{d}\left(K \cap B_{x, r}\right)-\mathcal{H}^{d}(D)\right)
$$

holds for every compact $d$-rectifiable set $K \subset \overline{B_{x, r}}$ such that $D \subset \pi_{T}(K)$, where $\pi_{T}: \mathbb{R}^{n} \rightarrow T$ is the orthogonal projection of $\mathbb{R}^{n}$ onto $T$.

Remark 1.4. Given a $d$-rectifiable set $K$ and a deformation $\varphi \in \mathrm{D}(x, r)$, using Property (1.1), we deduce the quasiminimality property

$$
\mathbf{F}(\varphi(K)) \leq \Lambda \mathcal{H}^{d}(\varphi(K)) \leq \Lambda(\operatorname{Lip}(\varphi))^{d} \mathcal{H}^{d}(K) \leq \frac{\Lambda}{\lambda}(\operatorname{Lip}(\varphi))^{d} \mathbf{F}(K)
$$

In DPDRG16] the authors obtained an extension of Allard's rectifiability Theorem for varifolds stationary with respect to anisotropic integrands. Let us first recall the following condition, introduced in [DPDRG16]:

Definition 1.5. For a given integrand $F \in C^{1}(\Omega \times G(n, d)), x \in \Omega$, and a Borel probability measure $\mu \in \mathcal{P}(G(n, d))$, let us define

$$
A_{x}(\mu):=\int_{G(n, d)} B_{F}(x, T) d \mu(T) \in \mathbb{R}^{n} \otimes \mathbb{R}^{n} .
$$

We say that $F$ verifies the atomic condition $(A C)$ at $x$ if the following two conditions are satisfied:

(i) $\operatorname{dim} \operatorname{ker} A_{x}(\mu) \leq n-d$ for all $\mu \in \mathcal{P}(G(n, d))$,

(ii) if $\operatorname{dim} \operatorname{ker} A_{x}(\mu)=n-d$, then $\mu=\delta_{T_{0}}$ for some $T_{0} \in G(n, d)$.

The following rectifiability criterion is the main result in [DPDRG16]:

Theorem 1.6 ([DPDRG16, Theorem 1.2]). Let $F \in C^{1}\left(G\left(\mathbb{R}^{n}\right), \mathbb{R}^{+}\right)$be a positive integrand satisfying the $(A C)$ condition, and let us suppose that $V$ is a d-dimensional varifold such that:

- $V$ has bounded anisotropic first variation: $\delta_{F} V$ is a Radon measure.

- $V$ has a lower density bound: there exists $\theta_{0}>0$ such that

$$
\frac{\|V\|\left(B_{x, r}\right)}{r^{d}}=\frac{V\left(B_{x, r} \times G(n, d)\right)}{r^{d}} \geq \theta_{0} \quad \text { for all } x \in K \text { and } r<\operatorname{dist}(x, H) .
$$


Then $V$ is d-rectifiable.

We can now state the main result of this paper:

Theorem 1.7. Let $F \in C^{1}\left(G\left(\mathbb{R}^{n}\right)\right)$ be an integrand satisfying (1.1), (1.5) and the $A C$ condition. Let $H \subset \mathbb{R}^{n}$ be closed and $\mathcal{P}(H)$ be a good class. Assume that the infimum in the Plateau problem (1.3) is finite and let $\left\{K_{j}\right\} \subset \mathcal{P}(H)$ be a minimizing sequence. Then, up to subsequences, the measures $\mu_{j}:=F\left(\cdot, T_{K_{j}}(\cdot)\right) \mathcal{H}^{d}\left\llcorner K_{j}\right.$ converge weakly in $\mathbb{R}^{n} \backslash H$ to the measure $\mu=F\left(\cdot, T_{K}(\cdot)\right) \mathcal{H}^{d}\llcorner K$, where $K=\operatorname{spt} \mu \backslash H$ is a d-rectifiable set. Furthermore, the integral varifold naturally associated to $\mu$ is F-stationary in $\mathbb{R}^{n} \backslash H$. In particular, $\liminf { }_{j} \mathbf{F}\left(K_{j}\right) \geq \mathbf{F}(K)$ and if $K \in \mathcal{P}(H)$, then $K$ is a minimum for (1.3).

Remark 1.8. Our proof of Theorem 1.7 strongly relies on Theorem 1.6 for which the $A C$ condition is essentially necessary, see the discussion in [DPDRG16]. In the case when $d=n-1$ (or if $d=1$ ) $A C$ condition is equivalent to the the strict convexity of $F$ and thus it implies (1.5) with $\Gamma=0$, see [DPDRG16, Theorem 1.3]. When $2 \leq d \leq(n-1)$ the situation is more subtle and a complete characterization is not known. However, in the recent work [DK] of the second author with S. Kolasinski (completed while the current one was submitted) it is shown that the $A C$ condition implies the validity of (1.5) with $\Gamma=0$, see [DK, Theorem 8.9]. Hence to prove Theorem 1.7 one can just assume that $F$ satisfies the $A C$ condition.

Remark 1.9. For the boundary conditions considered in [HP13] and in Dav14, Dav13], the corresponding classes $\mathcal{P}(H)$ are all good, as proved in the previous works [DGM14, DPDRG15, DLDRG16] . By the same arguments in these papers, one can also show that the set $K$ in Theorem [1.7 is actually a minimum for the formulation in [HP13], see [DPDRG15, Theorem 1.5] (here restricting our deformation to those which can be obtained as limit of orientation preserving diffeomorphism turns out to be crucial). In this paper we extend this analysis to provide a new proof of Reifenberg existence theorem, [Rei60], which does not rely on his topological disk theorem, see Section 3 for the precise setting and Theorem 3.4 there.

Remark 1.10. We observe that in case the set $K$ provided by the Theorem 1.7 belongs to $\mathcal{P}(H)$, it has minimal $\mathbf{F}$ energy with respect to deformations in the classes $\mathrm{D}(x, r)$ of Definition 1.1. with $x \in K$ and $H \cap B_{x, r}=\emptyset$. Although the union of these classes is strictly contained in the class of all Lipschitz deformations, it is rich enough to generate the comparison sets in [Alm76], Hence if one assumes that (1.5) is true with some $\Gamma>0$, one obtains almost everywhere regularity of $K$, see [Alm76, III.1 and III.3].

Acknowledgements. G.D.P. is supported by the MIUR SIR-grant Geometric Variational Problems (RBSI14RVEZ). A.D.R. is supported by SNF 159403 Regularity questions in geometric measure theory. F.G. is supported by the ERC Starting Grant FLIRT - Fluid Flows and Irregular Transport.

\section{Proof of Theorem 1.7}

The proof of Theorem 1.7 shares some arguments with the results in [DPDRG15]: in order to keep the outline simple we will sometimes refer to that paper for the common parts, while presenting the original content of the proof in detail.

We will use the following notations: $Q_{x, l}$ denotes the closed cube centered in $x$, with edge length $l$;

$$
R_{x, a, b}:=x+\left[-\frac{a}{2}, \frac{a}{2}\right]^{d} \times\left[-\frac{b}{2}, \frac{b}{2}\right]^{n-d}
$$

denotes the "rectangle" with sides $a$ and $b$, in the splitting $\mathbb{R}^{n}=\mathbb{R}^{d} \times \mathbb{R}^{n-d}$ (hence $Q_{x, l}=R_{x, l, l}$ ). Cubes and balls in the subspace $\mathbb{R}^{d} \times\{0\}^{n-d}$ are denoted by $Q_{x, l}^{d}$ and $B_{x, r}^{d}$, respectively. 
Proof of Theorem 1.7. Since the infimum in the Plateau problem (1.3) is finite, there exists a minimizing sequence $\left\{K_{j}\right\} \subset \mathcal{P}(H)$ and a Radon measure $\mu$ on $\mathbb{R}^{n} \backslash H$ such that

$$
\mu_{j} \stackrel{*}{\rightarrow} \mu, \quad \text { as Radon measures on } \mathbb{R}^{n} \backslash H,
$$

where $\mu_{j}=F\left(\cdot, T_{K_{j}}(\cdot)\right) \mathcal{H}^{d}\left\llcorner K_{j}\right.$. We set $K=\operatorname{spt} \mu \backslash H$ and consider also the canonical densityone rectifiable varifolds $V^{j}$ naturally associated to $K_{j}$ :

$$
V^{j}:=\mathcal{H}^{d}\left\llcorner K_{j} \otimes \delta_{T_{x} K_{j}} .\right.
$$

Since $K_{j}$ is a minimizing sequence in (1.3) and $F \geq \lambda$, for large $j$ we have the bound $\left\|V^{j}\right\|\left(\mathbb{R}^{n}\right) \leq$ $\frac{2 m_{0}}{\lambda}$ and therefore we can assume that $V^{j}$ converges to $V$ in the sense of varifolds.

Step 1: $V$ is F-stationary in $\mathbb{R}^{n} \backslash H$. Assume by contradiction the existence of $g \in C_{c}^{1}\left(\mathbb{R}^{n} \backslash H, \mathbb{R}^{n}\right)$ such that $\delta_{F} V(g)<0$. This information can be localized to a ball using a partition of unity for the compact set $\operatorname{spt}(\mathrm{g}) \subset \subset \mathbb{R}^{n} \backslash H$ : we can assume that for some ball $B_{x, r} \subset \subset \mathbb{R}^{n} \backslash H$ there is a vector field (not relabeled) $g \in C_{c}^{1}\left(B_{x, r}, \mathbb{R}^{n}\right)$ such that $\delta_{F} V(g)=:-2 c<0$. There exists $s>0$ such that $(\mathrm{Id}+t g) \in \mathrm{D}(x, r)$ for every $t \leq s$. By continuity of the functional $\delta_{F} Z(g)$ with respect to $Z$, up to consider a smaller $s>0$, it holds

$$
\delta_{F}(\mathrm{Id}+t g)^{\#} V(g) \leq-c<0, \quad \forall t \in[0, s] .
$$

Integrating the last inequality in $t$, we conclude that

$$
\mathbf{F}\left((\mathrm{Id}+s g)^{\#} V\right) \leq \mathbf{F}(V)-c s .
$$

Moreover there exists an open set $A$ with $B_{x, r} \subset A \subset \subset \mathbb{R}^{n} \backslash H$ and satisfying

$$
\|V\|(\partial A)=\left\|(\operatorname{Id}+s g)^{\#} V\right\|(\partial A)=0 .
$$

Since Id $+s g \in C_{c}^{1}(A)$, then (2.2) reads

$$
\mathbf{F}\left((\mathrm{Id}+s g)^{\#} V, A\right) \leq \mathbf{F}(V, A)-c s .
$$

Combining (2.3) with (2.4) , for $j$ large enough we infer

$$
\mathbf{F}\left((\operatorname{Id}+s g)^{\#} V^{j}, A\right) \leq \mathbf{F}\left(V^{j}, A\right)-c s+o_{j}, \quad \text { where } \lim _{j \rightarrow \infty} o_{j}=0,
$$

see also De 16, Section 5.1] for more details. Note that $\mathbf{F}\left((\operatorname{Id}+s g)^{\#} V^{j}, A\right)=\mathbf{F}\left((\operatorname{Id}+s g)\left(K_{j}\right), A\right)$ as well as $\mathbf{F}\left(V^{j}, A\right)=\mathbf{F}\left(K_{j}, A\right)$. Adding $\mathbf{F}\left(K_{j}, \mathbb{R}^{n} \backslash A\right)$ to both sides of (2.5) and observing that $(\mathrm{Id}+s g)\left(K_{j}\right) \backslash A=K_{j} \backslash A$, we obtain

$$
\mathbf{F}\left((\mathrm{Id}+s g)\left(K_{j}\right), \mathbb{R}^{n}\right) \leq \mathbf{F}\left(K_{j}, \mathbb{R}^{n}\right)-c s+o_{j}(1) .
$$

Since Id $+s g \in \mathrm{D}(x, r)$ and $B_{x, r} \subset \subset \mathbb{R}^{n} \backslash H$, by definition of good class, see Definition 1.2, there exists a new sequence $\left\{\tilde{K}_{j}\right\}_{j \in \mathbb{N}} \subseteq \mathcal{P}(H)$, such that

$$
\mathbf{F}\left(\tilde{K}_{j}\right) \leq \mathbf{F}\left((\operatorname{Id}+s g)\left(K_{j}\right)\right)+\frac{c s}{2}, \quad \forall j \in \mathbb{N} .
$$

Combining (2.6) with (2.7) and choosing $j$ big enough, we contradict the minimizing property of the sequence $\left\{K_{j}\right\}_{j \in \mathbb{N}}$.

Step 2: $V$ satisfies a density lower bound. We claim that there exists $\theta_{0}=\theta_{0}(n, d, \lambda, \Lambda)>0$ such that

$$
\|V\|\left(B_{x, r}\right) \geq \theta_{0} \omega_{d} r^{d}, \quad x \in \operatorname{spt}\|V\| \text { and } r<d_{x}:=\operatorname{dist}(x, H) .
$$

This can be achieved by the same techniques of [DPDRG15, Theorem 1.3, Step 1]. Indeed by (1.1) the integrand $F$ is comparable to the area and this is the only property needed in the proof in [DPDRG15], see also [De 16].

\footnotetext{
${ }^{1}$ Here $\|V\|$ is the projection of the measure $V$ on the first factor, i.e. $\|V\|(A)=V(A \times \mathbb{G})$ for every Borel set $A \subset \mathbb{R}^{d}$
} 
Step 3: $V$ is rectifiable. Combining the lower bound (2.8) with the $F$-stationarity in $\mathbb{R}^{n} \backslash H$ and applying Theorem [1.6 we conclude that $V$ is a $d$-rectifiable varifold and in turn that $\mu=$ $\mathbf{F}(V, \cdot)=\theta \mathcal{H}^{d}\left\llcorner\tilde{K}\right.$ for some countably $\mathcal{H}^{d}$-rectifiable set $\tilde{K}$ and some positive Borel function $\theta$. Since $K$ is the support of $\mu$, then $\mathcal{H}^{d}(\tilde{K} \backslash K)=0$. On the other hand, by differentiation of Hausdorff measures, (2.8) yields $\mathcal{H}^{d}(K \backslash \tilde{K})=0$. Hence $K$ is $d$-rectifiable and

$$
\mu=\theta \mathcal{H}^{d}\llcorner K \text {. }
$$

We now proceed to compute the exact value of the density $\theta$ : to this end we need the following elementary lemma, whose proof can be obtained as in [DLDRG16, Lemma 3.2].

Lemma 2.1. Let $K$ be the d-rectifiable set obtained in Step 3. For every $x$ where $K$ has an approximate tangent plane $T_{K}(x)$, let $O_{x}$ be a special orthogonal transformation of $\mathbb{R}^{n}$ mapping $\left\{x_{d+1}=\cdots=x_{n}=0\right\}$ onto $T_{K}(x)$ and set $\bar{Q}_{x, r}=O_{x}\left(Q_{x, r}\right)$ and $\bar{R}_{x, r, \varepsilon r}=O_{x}\left(R_{x, r, \varepsilon r}\right)$. At almost every $x \in K$ the following holds: for every $\varepsilon>0$ there exist $r_{0}=r_{0}(x) \leq \frac{1}{\sqrt{n+1}} \operatorname{dist}(x, H)$ such that, for $r \leq r_{0} / 2$,

$$
\begin{gathered}
\left(\theta_{0} \omega_{d}-\varepsilon\right) r^{d} \leq \mu\left(B_{x, r}\right) \leq\left(\theta(x) \omega_{d}+\varepsilon\right) r^{d}, \quad(\theta(x)-\varepsilon) r^{d}<\mu\left(\bar{Q}_{x, r}\right)<(\theta(x)+\varepsilon) r^{d}, \\
\sup _{y \in B_{x, r_{0}}, S \in G}|F(y, S)-F(x, S)| \leq \varepsilon,
\end{gathered}
$$

where $\theta_{0}=\theta_{0}(n, d, \lambda, \Lambda)$ is the universal lower bound obtained in (2.8). Moreover, for almost every such $r$, there exists $j_{0}(r) \in \mathbb{N}$ such that for every $j \geq j_{0}$ :

$$
\begin{gathered}
\left(\theta(x) \omega_{d}-\varepsilon\right) r^{d} \leq \mathbf{F}\left(K_{j}, B_{x, r}\right) \leq\left(\theta(x) \omega_{d}+\varepsilon\right) r^{d}, \\
(\theta(x)-\varepsilon) r^{d} \leq \mathbf{F}\left(K_{j}, \bar{Q}_{x, r}\right) \leq(\theta(x)+\varepsilon) r^{d}, \quad \mathbf{F}\left(K_{j}, \bar{Q}_{x, r} \backslash \bar{R}_{x, r, \varepsilon r}\right)<\varepsilon r^{d} .
\end{gathered}
$$

We are now ready to complete the proof of Theorem 1.7, namely to show $\liminf { }_{j} \mathbf{F}\left(K_{j}\right) \geq$ $\mathbf{F}(K)$ and that $\mu=F\left(x, T_{K}(x)\right) \mathcal{H}^{d}\llcorner K$.

Step 4: $\theta(x) \geq F\left(x, T_{K}(x)\right)$ for almost every $x \in K$. We prove that $\theta(x) \geq F\left(x, T_{K}\right)$ for every $x \in K$ that satisfies the properties of Lemma 2.1 (thus, $\mathcal{H}^{d}$-a.e. on $K$ ). Let us fix $\varepsilon<r_{0} / 2$ and choose a radius $r<r_{0} / 2$ such that both $r$ and $(1-\sqrt{\varepsilon}) r$ satisfy properties (2.10)-(2.13) and $\mu\left(\partial Q_{r}\right)=0$. Let us assume without loss of generality that $x=0$ and $T_{K}=\left\{x_{d+1}=\ldots=x_{n}=\right.$ $0\}$. Since the $\mu_{j}$ are weakly converging to $\mu$, we get that for $j \geq j(r)$

$$
\mu_{j}\left(Q_{r} \backslash R_{r, \varepsilon r}\right)=o_{j}(1) .
$$

First of all we deform $K_{j}$ so that the mass in (2.14) is zero: by repeating verbatim the argument in [DPDRG15, Theorem 1.3, Step 4], we construct a map $\psi \in \mathrm{D}(0,2 r)$ such that, setting $\widetilde{K}_{j}:=\psi\left(K_{j}\right)$, we have (see [DPDRG15, Equation (3.16)])

$$
\mathcal{H}^{d}\left(\widetilde{K_{j}} \cap\left(Q_{r(1-\sqrt{\varepsilon})} \backslash R_{r(1-\sqrt{\varepsilon}), 6 \varepsilon r}\right)\right)=0,
$$

and consequently (see DPDRG15, Equation (3.18)])

$$
\mathbf{F}\left(K_{j}, B_{r(1-\sqrt{\varepsilon})}\right) \geq \mathbf{F}\left(\widetilde{K}_{j}, B_{r(1-\sqrt{\varepsilon})}\right)-C \sqrt{\varepsilon} r^{d} .
$$

We claim that

$$
B_{r(1-\sqrt{\varepsilon})} \cap T_{K} \subset \pi\left(\widetilde{K_{j}} \cap R_{r(1-\sqrt{\varepsilon}), 6 \varepsilon r}\right)
$$

where $\pi: \mathbb{R}^{n} \rightarrow T_{K}$ is the orthogonal projection of $\mathbb{R}^{n}$ onto $T_{K}$. Note that this immediately implies that

$$
\overline{B_{r(1-\sqrt{\varepsilon})}} \cap T_{K} \subset \pi\left(\widetilde{K_{j}} \cap \overline{B_{r(1-\sqrt{\varepsilon})}}\right) .
$$

Therefore the ellipticity of $F$ (namely (1.5)), (2.11), (2.12), (1.6) and (2.8) imply that

$$
\mathbf{F}^{0}\left(T_{K}, B_{r(1-\sqrt{\varepsilon})}\right) \leq \mathbf{F}^{0}\left(\widetilde{K_{j}}, B_{r(1-\sqrt{\varepsilon})}\right) \leq \mathbf{F}\left(\widetilde{K_{j}}, B_{r(1-\sqrt{\varepsilon})}\right)+C \varepsilon r^{d},
$$


hence

$$
\begin{aligned}
\theta(0) \omega_{d} r^{d}(1-\sqrt{\varepsilon}) & \frac{d}{\stackrel{(2.12)}{\geq}} \mathbf{F}\left(K_{j}, B_{r(1-\sqrt{\varepsilon})}\right)-\varepsilon r^{d} \stackrel{(2.16)}{\geq} \mathbf{F}\left(\widetilde{K_{j}}, B_{r(1-\sqrt{\varepsilon})}\right)-C \sqrt{\varepsilon} r^{d} \\
& \stackrel{(2.18)}{\geq} \mathbf{F}^{0}\left(T_{K}, B_{r(1-\sqrt{\varepsilon})}\right)-C \sqrt{\varepsilon} r^{d}=F\left(0, T_{K}\right) \omega_{d} r^{d}(1-\sqrt{\varepsilon})^{d}-C \sqrt{\varepsilon} r^{d}
\end{aligned}
$$

which yields $\theta(0) \geq F\left(0, T_{K}\right)$.

We are thus left to prove (2.17). To this end, as in [DPDRG15, Theorem 1.3, Step 4], we extend $\pi_{\mid R_{r(1-\sqrt{\varepsilon}), 6 \varepsilon r}}$ to a map $P \in \mathrm{D}(0,2 r)$ that collapses $R_{r(1-\sqrt{\varepsilon}), 6 \varepsilon r}$ onto $T_{K}$, is the identity outside $Q_{r}$ and satisfies $\operatorname{Lip} P \leq 1+C \sqrt{\varepsilon}$ for some dimensional constant $C$.

We now set $K_{j}^{\prime}:=P\left(\widetilde{K}_{j}\right)=(P \circ \psi)\left(K_{j}\right)$. Thanks to (2.15),

$$
\mathcal{H}^{d}\left(K_{j}^{\prime} \cap\left(Q_{(1-\sqrt{\varepsilon}) r} \backslash Q_{(1-\sqrt{\varepsilon}) r}^{d}\right)\right)=0 ;
$$

furthermore the estimates (2.13) and (1.6) imply also

$$
\mathbf{F}\left(K_{j}^{\prime}, Q_{r} \backslash Q_{r(1-\sqrt{\varepsilon})}\right) \leq C(\Lambda, \lambda, P) \mathbf{F}\left(K_{j}, Q_{r} \backslash Q_{r(1-\sqrt{\varepsilon})}\right) \leq C \sqrt{\varepsilon} r^{d} .
$$

Assume now by contradiction that (2.17) fails. Since $K_{j}^{\prime}$ is closed and $P=\pi$ in $R_{r(1-\sqrt{\varepsilon}), 6 \varepsilon r}$, there would exist $y_{j}^{\prime} \in Q_{(1-\sqrt{\varepsilon}) r}^{d}$ and $\delta_{j}>0$ such that, if we set $y_{j}:=\left(y_{j}^{\prime}, 0\right)$, then

$$
K_{j}^{\prime} \cap B_{y_{j}, \delta_{j}}^{d}=\emptyset \quad \text { and } \quad B_{y_{j}, \delta_{j}}^{d} \subset Q_{(1-\sqrt{\varepsilon}) r}^{d} .
$$

We now consider the deformation map $\varphi_{j}$ retracting $Q_{(1-\sqrt{\varepsilon}) r}^{d} \backslash B_{y_{j}, \delta_{j}}^{d}$ onto the equator $\partial Q_{(1-\sqrt{\varepsilon}) r}^{d}$ and not moving $Q_{(1-\sqrt{\varepsilon}) r}^{c}$, constructed in [DPDRG15, Theorem 1.3, Step 4]. In particular, letting $K_{j}^{\prime \prime}:=\varphi_{j}\left(K_{j}^{\prime}\right)$, the following properties are satisfied:

$$
\varphi_{j}\left(Q_{(1-\sqrt{\varepsilon}) r}^{d}\right) \subset \partial Q_{(1-\sqrt{\varepsilon}) r}^{d}
$$

and

$$
K_{j}^{\prime \prime} \backslash Q_{r}=K_{j} \backslash Q_{r}
$$

Thanks to (2.19) we obtain:

$$
\mathcal{H}^{d}\left(K_{j}^{\prime \prime} \cap Q_{r(1-\sqrt{\varepsilon})}\right)=0 .
$$

By the very definition of $\mathcal{P}(H)$ we can find $J_{j} \in \mathcal{P}(H), \varepsilon r^{d}$-close in energy to $K_{j}^{\prime \prime}$. Since $K_{j}^{\prime}$ and $K_{j}^{\prime \prime}$ coincide outside $Q_{(1-\sqrt{\varepsilon}) r}$, we then conclude that:

$$
\begin{aligned}
\mathbf{F}\left(K_{j}\right)-\mathbf{F}\left(J_{j}\right) & \geq \mathbf{F}\left(K_{j}, Q_{r}\right)-\mathbf{F}\left(K_{j}^{\prime \prime}, Q_{r}\right)-\varepsilon r^{d} \\
& \stackrel{(2.22)}{\geq} \mathbf{F}\left(K_{j}, Q_{r(1-\sqrt{\varepsilon})}\right)+\mathbf{F}\left(K_{j}, Q_{r} \backslash Q_{r(1-\sqrt{\varepsilon})}\right)-\mathbf{F}\left(K_{j}^{\prime}, Q_{r} \backslash Q_{r(1-\sqrt{\varepsilon})}\right)-\varepsilon r^{d} \\
& \stackrel{(2.20)}{\geq}(\theta(0)-\varepsilon)(1-\sqrt{\varepsilon})^{d} r^{d}-C \sqrt{\varepsilon} r^{d} \geq(\theta(0)-C \sqrt{\varepsilon}) r^{d}>0 .
\end{aligned}
$$

Since this is in contradiction with the minimizing property of the sequence $\left\{K_{j}\right\}$, we conclude the proof of (2.17).

Step 5: $\theta(x) \leq F\left(x, T_{K}(x)\right)$ for almost every $x \in K$ : Again we assume that $x=0$ is a point satisfying the conclusion of Lemma 2.1 and we argue as in DPDRG15, Theorem 1.3, Step 5].

Arguing by contradiction, we assume that $\theta(0)=F\left(0, T_{K}(0)\right)+\sigma$ for some $\sigma>0$ and let $\varepsilon<\min \left\{\frac{\sigma}{2}, \frac{\lambda \sigma}{4 \Lambda}\right\}$. As a consequence of (2.13), there exist $r$ and $j_{0}=j_{0}(r)$ such that

$$
\mathbf{F}\left(K_{j}, Q_{r}\right)>\left(F(0, T)+\frac{\sigma}{2}\right) r^{d}, \quad \mathbf{F}\left(K_{j}, Q_{r} \backslash R_{r, \varepsilon r}\right)<\frac{\lambda \sigma}{4 \Lambda} r^{d}, \quad \forall j \geq j_{0} .
$$


Consider the map $P \in \mathrm{D}(0, r)$ defined in [DPDRG15, Equation 3.14] which collapses $R_{r(1-\sqrt{\varepsilon}), \varepsilon r}$ onto the tangent plane $T_{K}$ and satisfies $\|P-I d\|_{\infty}+\operatorname{Lip}(P-I d) \leq C \sqrt{\varepsilon}$. Exploiting the fact that $\mathcal{P}(H)$ is a good class and by almost minimality of $K_{j}$, we find that

$$
\begin{aligned}
\mathbf{F}\left(K_{j}, Q_{r}\right)-o_{j}(1) & \leq \underbrace{\mathbf{F}\left(P\left(K_{j}\right), P\left(R_{(1-\sqrt{\varepsilon}) r, \varepsilon r}\right)\right)}_{I_{1}}+\underbrace{\mathbf{F}\left(P\left(K_{j}\right), P\left(R_{r, \varepsilon r} \backslash R_{(1-\sqrt{\varepsilon}) r, \varepsilon r}\right)\right)}_{I_{2}} \\
& +\underbrace{\mathbf{F}\left(P\left(K_{j}\right), P\left(Q_{r} \backslash R_{r, \varepsilon r}\right)\right)}_{I_{3}} .
\end{aligned}
$$

By the properties of $P$ and (2.11), we get $I_{1} \leq\left(F\left(0, T_{K}\right)+\varepsilon\right) r^{d}$, while, by (2.23) and equation (1.6)

$$
I_{3} \leq \frac{\Lambda}{\lambda}(\operatorname{Lip} P)^{d} \mathbf{F}\left(K_{j}, Q_{r} \backslash R_{r, \varepsilon r}\right)<(1+C \sqrt{\varepsilon})^{d} \frac{\sigma}{4} r^{d} .
$$

Since $\mathbf{F}\left(P\left(K_{j}\right), P\left(R_{r, \varepsilon r} \backslash R_{(1-\sqrt{\varepsilon}) r, \varepsilon r}\right)\right) \leq \frac{\Lambda}{\lambda}(1+C \sqrt{\varepsilon})^{d} \mathbf{F}\left(K_{j}, R_{r, \varepsilon r} \backslash R_{(1-\sqrt{\varepsilon}) r, \varepsilon r}\right)$ and $R_{r, \varepsilon r} \backslash$ $R_{(1-\sqrt{\varepsilon}) r, \varepsilon r} \subset Q_{(1-\sqrt{\varepsilon}) r} \backslash Q_{r}$, by (2.13) we can also bound

$$
\begin{aligned}
I_{2}=\mathbf{F}( & \left.P\left(K_{j}\right), P\left(R_{r, \varepsilon r} \backslash R_{(1-\sqrt{\varepsilon}) r, \varepsilon r}\right)\right) \leq \frac{\Lambda}{\lambda}(1+C \sqrt{\varepsilon})^{d} \mathbf{F}\left(K_{j}, Q_{r} \backslash Q_{(1-\sqrt{\varepsilon}) r}\right) \\
& \leq C(1+C \sqrt{\varepsilon})^{d}\left(\left(F\left(0, T_{K}\right)+\sigma+\varepsilon\right)-\left(F\left(0, T_{K}\right)+\sigma-\varepsilon\right)(1-\sqrt{\varepsilon})^{d}\right) r^{d} \leq C \sqrt{\varepsilon} r^{d} .
\end{aligned}
$$

Hence, as $j \rightarrow \infty$, by (2.10)

$$
\left(F\left(0, T_{K}\right)+\frac{\sigma}{2}\right) r^{d} \leq\left(F\left(0, T_{K}\right)+\varepsilon\right) r^{d}+C \sqrt{\varepsilon} r^{d}+(1+C \sqrt{\varepsilon})^{d} \frac{\sigma}{4} r^{d}:
$$

dividing by $r^{d}$ and letting $\varepsilon \downarrow 0$ provides the desired contradiction.

Combining (2.9) with Step 4 and Step 5, we conclude the proof of the theorem.

\section{Solution of the Plateau problem in the Reifenberg's formulation}

The Reifenberg formulation of the Plateau problem in [Rei60] involves an algebraic notion of boundary described in terms of Čech homology groups. The particular choice of an homology theory defined on compact spaces and with coefficient groups that are abelian and compact has three motivations:

(i) These assumptions imply that the homology groups are continuous with respect to the Hausdorff convergence of the spaces.

(ii) It satisfies the classical axioms of Eilenberg and Steenrod, enabling the use of the MayerVietoris exact sequence, ES52, Chapter X].

(iii) It ensures the crucial property

$$
\mathcal{H}^{\ell}(K)=0 \Rightarrow \check{H}_{\ell}(K)=0,
$$

which may fail in general for other homology theories, [BM62].

In this section we follow Reifenberg's approach and show that we can obtain a minimizing set in the chosen homology class.

Let $\mathbb{G}$ be a compact Abelian group and let $K$ be a closed set in $\mathbb{R}^{n}$. For every $m \geq 0$ we denote with $\check{H}_{m}(K ; \mathbb{G})$ (often omitting the explicit mention of the group $\mathbb{G}$ ) the $m^{\text {th }}$-Cech homology group of $K$ with coefficients in $\mathbb{G}$, ES52, Chapter IX].

Recall that, if $H \subset K$ is a compact set, the inclusion map $i_{H, K}: H \rightarrow K$ induces a graded homomorphism between the homology groups (of every grade $m$, again often omitted)

$$
i_{* H, K}: \check{H}_{m}(H, \mathbb{G}) \rightarrow \check{H}_{m}(K, \mathbb{G}) .
$$

Note, in the next definition, the role of the dimension $d$, inherent of our variational problem. 
Definition 3.1 (Boundary in the sense of Reifenberg). Let $\mathbb{G}, H, K$ be as above and let $L \subset$ $\check{H}_{d-1}(H, \mathbb{G})$ be a subgroup. We say that $K$ has boundary $L$ if

$$
\operatorname{Ker}\left(i_{* H, K}\right) \supset L \text {. }
$$

Definition 3.2 (Reifenberg class). Let $\mathbb{G}, H, K, L$ be as above; we let $\mathcal{R}(H)$ be the class of closed $d$-rectifiable subsets $K$ of $\mathbb{R}^{n} \backslash H$ contained in the same ball $B \ni H$ and such that $K \cup H$ has boundary $L$ in the sense of Definition 3.1 .

Remark 3.3. We remark that $\mathcal{R}(H)$ is a good class in the sense of Definition 1.2, Indeed for every $K \in \mathcal{R}(H)$, every $x \in K, r \in(0, \operatorname{dist}(x, H))$ and $\varphi \in \mathrm{D}(x, r)$ (which is in particular continuous),

$$
\varphi(K \cup H)=\varphi(K) \cup H
$$

and moreover by functoriality $\operatorname{Ker}\left(\varphi \circ i_{H, K \cup H}\right)_{*} \supset \operatorname{Ker}\left(i_{* H, K \cup H}\right) \supset L$, which implies that $\varphi(K) \in \mathcal{R}(H)$. We can therefore apply Theorem 1.7 to the class $\mathcal{R}(H)$ to obtain the existence of a relatively closed subset $K$ of $\mathbb{R}^{n} \backslash H$ satisfying

$$
\mathbf{F}(K)=\inf _{S \in \mathcal{R}(H)}\{\mathbf{F}(S)\} .
$$

We address now the question of whether $K$ belongs to the Reifenberg class $\mathcal{R}(H)$. Recall the definition of Hausdorff distance between two compact sets $C_{1}, C_{2} \subset \mathbb{R}^{n}$ :

$$
d_{\mathcal{H}}\left(C_{1}, C_{2}\right):=\inf \left\{r>0: C_{1} \subset U_{r}\left(C_{2}\right) \text { and } C_{2} \subset U_{r}\left(C_{1}\right)\right\},
$$

where we denote with $U_{r}(C)$ the $r$-neighborhood of $C$.

The main result of this section is the following theorem:

Theorem 3.4. For every minimizing sequence $\left\{K_{j}\right\} \subset \mathcal{R}(H)$ the associated limit set given by Theorem 1.7 satisfies $K \in \mathcal{R}(H)$.

The proof of the above theorem will be obtained by constructing another minimizing sequence, $\left(K_{j}^{1}\right) \subset \mathcal{R}(H)$, yielding the same limit set $K$, but with the further property that

$$
d_{\mathcal{H}}\left(K_{j}^{1} \cup H, K \cup H\right) \rightarrow 0 .
$$

To do this, we will first deform the part of $K_{k}$ lying outside an $\varepsilon_{j}$-neighbourhood $U_{\varepsilon_{j}}(K)\left(\varepsilon_{j} \rightarrow 0\right.$, $k \gg j$ ) onto a $(d-1)$-skeleton and then take its intersection with $U_{\varepsilon_{j}}(K)$. An application of the Mayer-Vietoris sequence will yield that the new sequence still satisfies the homological boundary conditions, see Step 4 below.

In turn, this last step relies on the properties (ii) and (iii) above. As in the case of the work by Reifenberg [Rei60], this forces us to restrict ourselves to compact coefficient groups. Let us remark that the extension of Reifenberg theorem to $\mathbb{Z}$ coefficients has been given in [Fan16] with different techniques.

Proof. Step 1: Construction of the new sequence. From Theorem 1.7 we know that $\mu_{k}:=$ $F\left(\cdot, T_{(\cdot)} K_{k}\right) \mathcal{H}^{d}\left\llcorner K_{k}\right.$ converge weakly ${ }^{\star}$ in $\mathbb{R}^{n} \backslash H$ to the measure $\mu=F\left(\cdot, T_{(\cdot)} K\right) \mathcal{H}^{d}\llcorner K$. Then, for every $\varepsilon_{j}>0$, there exists $k_{j}$ big enough so that

$$
\mu_{k_{j}}\left(\mathbb{R}^{n} \backslash U_{\varepsilon_{j}}(K \cup H)\right)<\frac{\Lambda \varepsilon_{j}^{d}}{k_{1}(4 n)^{d}},
$$

with $\Lambda$ the constant in equation (1.1) and $k_{1}$ is the constant appearing in the Deformation Theorem, DPDRG15, Theorem 2.4].

We cover $U_{5 \varepsilon_{j}}(K \cup H) \backslash U_{2 \varepsilon_{j}}(K \cup H)$ with a complex $\Delta$ of closed cubes with side length equal to $\varepsilon_{j} /(4 n)$ contained in $U_{6 \varepsilon_{j}}(K \cup H) \backslash U_{\varepsilon_{j}}(K \cup H)$. We can apply an adaptation of the Deformation Theorem [DPDRG15, Theorem 2.4] relative to the set $K_{k_{j}}$ and obtain a Lipschitz 
deformation $\varphi_{j}:=\varphi_{\varepsilon_{j} / 4 n, K_{k_{j}}}$. Observe that $\varphi_{j}\left(K_{k_{j}}\right) \cap\left(U_{4 \varepsilon_{j}}(K \cup H) \backslash U_{3 \varepsilon_{j}}(K \cup H)\right) \subset \Delta_{d}$, the $d$-skeleton of the complex. We claim that

$$
\varphi_{j}\left(K_{k_{j}}\right) \cap\left(U_{4 \varepsilon_{j}}(K \cup H) \backslash U_{3 \varepsilon_{j}}(K \cup H)\right) \subset \Delta_{d-1} .
$$

Otherwise by [DPDRG15, Theorem 2.4] point (5), $\varphi_{j}\left(K_{k_{j}}\right) \cap\left(U_{4 \varepsilon_{j}}(K \cup H) \backslash U_{3 \varepsilon_{j}}(K \cup H)\right)$ should contain an entire $d$-face of edge length $\varepsilon_{j} / 4 n$, leading together with (3.3) to a contradiction:

$$
\begin{aligned}
\frac{\varepsilon_{j}^{d}}{(4 n)^{d}} & \leq \mathcal{H}^{d}\left(\varphi_{j}\left(K_{k_{j}}\right) \cap\left(U_{4 \varepsilon_{j}}(K \cup H) \backslash U_{3 \varepsilon_{j}}(K \cup H)\right)\right) \leq k_{1} \mathcal{H}^{d}\left(K_{k_{j}} \backslash U_{\varepsilon_{j}}(K \cup H)\right) \\
& \leq \frac{k_{1}}{\lambda} \mathbf{F}\left(K_{k_{j}} \backslash U_{\varepsilon_{j}}(K \cup H)\right) \leq \frac{k_{1}}{\lambda} \mu_{k_{j}}\left(\mathbb{R}^{n} \backslash U_{\varepsilon_{j}}(K \cup H)\right)<\frac{\varepsilon_{j}^{d}}{(4 n)^{d}} .
\end{aligned}
$$

Set $\widetilde{K_{j}}:=\varphi_{j}\left(K_{k_{j}}\right)$ : by (3.4) and the coarea formula [Fed69, 3.2.22(3)], there exists $\alpha_{j} \in(3,4)$ such that

We now let

$$
\mathcal{H}^{d-1}\left(\widetilde{K_{j}} \cap \partial U_{\alpha_{j} \varepsilon_{j}}(K \cup H)\right)=0
$$

$$
K_{j}^{1}:=\widetilde{K_{j}} \cap \overline{U_{\alpha_{j} \varepsilon_{j}}(K \cup H)} \text { and } \quad K_{j}^{2}:=\widetilde{K}_{j} \backslash U_{\alpha_{j} \varepsilon_{j}}(K \cup H) .
$$

Step 2: proof of the property (3.2).

Note that by construction,

$$
K_{j}^{1} \cup H \subset U_{4 \varepsilon_{j}}(K \cup H), \quad \forall j \in \mathbb{N} .
$$

If on the other hand for some positive $\bar{\varepsilon}$ there is $x \in K \cup H \backslash U_{\bar{\varepsilon}}\left(K_{j}^{1} \cup H\right)$, then necessarily $d(x, H) \geq \bar{\varepsilon}$ as well as $d\left(x, K_{j}^{1}\right) \geq \bar{\varepsilon}$ : the weak convergence $\mu_{k_{j}} \stackrel{*}{\rightarrow} \mu$ would imply that

$$
\mu(B(x, \bar{\varepsilon} / 2)) \leq \liminf _{j \rightarrow \infty} \mu_{k_{j}}(B(x, \bar{\varepsilon} / 2))=0,
$$

which contradicts the uniform density lower bounds (2.8) on $B(x, \bar{\varepsilon} / 2)$. This yields (3.2).

Step 3: boundary constraint of the new sequence. To conclude the proof of Theorem 3.4, we need to check that $\left(K_{j}^{1}\right) \subset \mathcal{R}(H)$. By (3.6) we get

$$
\widetilde{K_{j}}=K_{j}^{1} \cup K_{j}^{2}, \quad \text { and } \quad K_{j}^{1} \cap K_{j}^{2}=\widetilde{K_{j}} \cap \partial U_{\alpha_{j} \varepsilon_{j}}(K)
$$

and (3.5), (3.6) yield

$$
\mathcal{H}^{d-1}\left(\left(K_{j}^{1} \cup H\right) \cap K_{j}^{2}\right)=\mathcal{H}^{d-1}\left(K_{j}^{1} \cap K_{j}^{2}\right)=0 .
$$

Therefore by [HW41, Theorem VIII 3']:

$$
\check{H}_{d-1}\left(\left(K_{j}^{1} \cup H\right) \cap K_{j}^{2}\right)=(0) .
$$

We furthermore observe that the sets $\widetilde{K_{j}}$ are obtained as deformations via Lipschitz maps strongly approximable via isotopies, and therefore belong to $\mathcal{R}(H)$. Since the map $\varphi_{j}$ coincides with the identity on $H$, we have

$$
i_{H, \widetilde{K_{j}} \cup H}=\varphi_{j} \circ i_{H, K_{j} \cup H}
$$

moreover, trivially $i_{H, \widetilde{K_{j}} \cup H}=i_{K_{j}^{1} \cup H, \widetilde{K_{j}} \cup H} \circ i_{H, K_{j}^{1} \cup H}$. Hence by functoriality

$$
\operatorname{Ker}\left(i_{* K_{j}^{1} \cup H, \widetilde{K_{j}} \cup H} \circ i_{* H, K_{j}^{1} \cup H}\right)=\operatorname{Ker}\left(i_{* H, \widetilde{K_{j}} \cup H}\right)=\operatorname{Ker}\left(\left(\varphi_{j}\right)_{*} \circ i_{* H, K_{j} \cup H}\right) \supset L .
$$

We claim that $i_{* K_{j}^{1} \cup H, \widetilde{K_{j}} \cup H}$ is injective: this implies that

$$
\operatorname{Ker}\left(i_{* H, K_{j}^{1} \cup H}\right) \supset L,
$$

namely $\left(K_{j}^{1}\right) \subset \mathcal{R}(H)$. 
Step 4: the map $i_{* K_{j}^{1} \cup H, \widetilde{K_{j}} \cup H}$ is injective. We can write the Mayer-Vietoris sequence (which for the Čech homology holds true for compact spaces and with coefficients in a compact group, due to the necessity of having the excision axiom, [ES52, Theorem 7.6 p.248]) and use (3.8):

(0) $\stackrel{\stackrel{3.8}{=}}{=} \check{H}_{d-1}\left(\left(K_{j}^{1} \cup H\right) \cap K_{j}^{2}\right) \stackrel{f}{\longrightarrow} \check{H}_{d-1}\left(K_{j}^{1} \cup H\right) \oplus \check{H}_{d-1}\left(K_{j}^{2}\right) \stackrel{g}{\longrightarrow} \check{H}_{d-1}\left(\widetilde{K}_{j} \cup H\right) \longrightarrow \ldots$

where $f=\left(i_{*\left(K_{j}^{1} \cup H\right) \cap K_{j}^{2}, K_{j}^{1} \cup H}, i_{*\left(K_{j}^{1} \cup H\right) \cap K_{j}^{2}, K_{j}^{2}}\right)$ and $g(\sigma, \tau)=\sigma-\tau$. The exactness of the sequence implies that $g$ is injective: in particular the map $g$ is injective when restricted to the subgroup $\check{H}_{d-1}\left(K_{j}^{1} \cup H\right) \oplus(0)$, where it coincides with $i_{* K_{j}^{1} \cup H, \widetilde{K_{j}} \cup H}$.

Step 5: boundary constraint for the limit set. Setting

$$
Y_{n}:=\overline{\bigcup_{j \geq n} K_{j}^{1} \cup H}
$$

by (3.2) we get

$$
d_{\mathcal{H}}\left(Y_{n}, K \cup H\right) \rightarrow 0
$$

Therefore $K \cup H$ is the inverse limit of the sequence $Y_{n}$. Since the sets $\left(K_{j}^{1} \cup H\right)$ are in the Reifenberg class $\mathcal{R}(H)$, namely the inclusion (3.9) holds, by composing the two injections $i_{* K_{j}^{1} \cup H, Y_{n}}$ and $i_{* H, K_{j}^{1} \cup H}$ for every $j \geq n$, we obtain that

$$
L \subset \operatorname{Ker}\left(i_{* H, Y_{n}}\right) .
$$

Since the Čech homology with coefficients in compact groups is continuous ES52, Definition 2.3], the latter inclusion is stable under Hausdorff convergence, [ES52, Theorem 3.1] (see also [Rei60, Lemma 21A]): therefore, by (3.10), we conclude

$$
L \subset \operatorname{Ker}\left(i_{* H, K \cup H}\right),
$$

and eventually $K \in \mathcal{R}(H)$.

Remark 3.5. Using the contravariance of cohomology theory, the same results can be obtained when considering a cohomological definition of boundary, again in the Čech theory, as introduced in [HP16]. In particular a new proof the the theorem there can be obtained with our assumption on the Lagrangian.

Note that in the cohomological definition of boundary all the Eilenberg-Steenrod axioms are satisfied even with a non-compact group $\mathbb{G}$. This allows us to consider as coefficients set the natural group $\mathbb{Z}$.

Remark 3.6. We observe that any minimizer $K$ as in Theorem 3.4 is also an $(\mathbf{F}, 0, \infty)$ minimal set in the sense of [Alm76, Definition III.1]. Indeed the boundary condition introduced in Definition 3.1 is preserved under Lipschitz maps (not necessarily in $\mathrm{D}(x, r)$ ). In particular, by Alm76, Theorem III.3(7)], if $F$ is smooth and strictly elliptic ( $\Gamma$ in Definition 1.3 is strictly positive), then $K$ is smooth away from the boundary, outside of a relative closed set of $\mathcal{H}^{d_{-}}$ measure zero.

\section{BIBLIOGRAPHY}

[All72] W. K. Allard. On the first variation of a varifold. Ann. Math., 95:417-491, 1972.

[Alm68] F. J. Jr Almgren. Existence and regularity almost everywhere of solutions to elliptic variational problems among surfaces of varying topological type and singularity structure. Ann. Math., 87:321-391, 1968.

[Alm76] F. J. Jr. Almgren. Existence and regularity almost everywhere of solutions to elliptic variational problems with constraints. Mem. Amer. Math. Soc., 4(165): viii+199 pp, 1976. 
[BM62] M. G. Barratt and John Milnor. An example of anomalous singular homology. Proc. Amer. Math. Soc., 13:293-297, $1962 . \quad$ ISSN 00029939. URL \protect \vrulewidth0pt \protect \href $\{$ http://dx.doi.org/10. 2307/2034486\} \{http://dx.doi.org/10.2307/2034486\}.

[Dav13] G. David. Regularity of minimal and almost minimal sets and cones: J. Taylor's theorem for beginners. In Analysis and geometry of metric measure spaces, volume 56 of CRM Proc. Lecture Notes, pages 67-117. Amer. Math. Soc., Providence, RI, 2013.

[Dav14] G. David. Should we solve Plateau's problem again? In Advances in Analysis: the legacy of Elias M. Stein. Princeton University Press, 2014.

[De 08] C. De Lellis. Rectifiable sets, densities and tangent measures. Zurich Lectures in Advanced Mathematics. European Mathematical Society, Zürich, 2008. vi+127 pp.

[DGM14] C. De Lellis, F. Ghiraldin, and F. Maggi. A direct approach to Plateau's problem. JEMS, 2014.

[DLDRG16] C. De Lellis, A. De Rosa, and F. Ghiraldin. A direct approach to the anisotropic Plateau problem. Accepted by Advances in Calculus of Variations, 2017 Available on arxiv: https://arxiv.org/abs/1602.08757. DOI: https://doi.org/10.1515/acv2016-0057

[DPDRG15] G. De Philippis, A. De Rosa, and F. Ghiraldin. A direct approach to Plateau's problem in any codimension. Adv. in Math., 288:59-80, January 2015.

[DPDRG16] G. De Philippis, A. De Rosa, and F. Ghiraldin. Rectifiability of varifolds with locally bounded first variation with respect to anisotropic surface energies. Comm. Pure Appl. Math., 71(6):1123-1148, 2016.

[De 16] A. De Rosa. Minimization of anisotropic energies in classes of rectifiable varifolds. SIAM Journal on Mathematical Analysis, 50(1):162-181, 2018.

[DK] A. De Rosa and S. Kolasinski. Equivalence of the ellipticity conditions for geometric variational problems. Available on arXiv: https://arxiv.org/abs/1810.07262, 2018.

[ES52] S. Eilenberg and N. Steenrod. Foundations of Algebraic Topology. Princeton University Press, 1952. 325-328 pp.

[Fan16] Y. Fang. Existence of minimizers for the Reifenberg plateau problem. Ann. Sc. Norm. Super. Pisa Cl. Sci. (5), 16(3):817-844, 2016.

[Fed69] H. Federer. Geometric measure theory, volume 153 of Die Grundlehren der mathematischen Wissenschaften. Springer-Verlag New York Inc., New York, 1969. xiv+676 pp.

[FK17] Y. Fang and S. Kolasiński. Existence of solutions to a general geometric elliptic variational problem. ArXiv e-prints, April 2017.

[HP13] J. Harrison and H. Pugh. Existence and soap film regularity of solutions to Plateau's problem. 2013. arXiv:1310.0508.

[HP15] J. Harrison and H. Pugh. Solutions To Lipschitz Variational Problems With Cohomological Spanning Conditions. 2015. arXiv:1506.01692.

[HP16] J. Harrison and H. Pugh. General Methods of Elliptic Minimization . 2016. https://arxiv.org/abs/1603.04492.

[HW41] W. Hurewicz and H. Wallman. Dimension Theory. Princeton Mathematical Series, v. 4. Princeton University Press, Princeton, N. J., 1941. vii+165 pp.

[Mor66] C. B. Jr Morrey. Multiple integrals in the calculus of variations. Springer-Verlag, Berlin Heidelberg New York, 1966.

[Pre87] D. Preiss. Geometry of measures in $\mathbf{R}^{n}$ : distribution, rectifiability, and densities. Ann. of Math. (2), 125(3):537-643, 1987.

[Rei60] E. R. Reifenberg. Solution of the Plateau problem for $m$-dimensional surfaces of varying topological type. Acta Math., 104:1-92, 1960. 
[Sim83] L. Simon. Lectures on geometric measure theory, volume 3 of Proceedings of the Centre for Mathematical Analysis. Australian National University, Centre for Mathematical Analysis, Canberra, 1983. vii+272 pp.

SISSA, Via Bonomea 265, 34136 Trieste, Italy

E-mail address: gdephili@sissa.it

Institut für Mathematik, Universität Zürich, Winterthurerstrasse 190, CH-8057 Zürich, SwitzerLAND

E-mail address: antonio.derosa@math.uzh.ch

Universität Basel, Spiegelgasse 1, CH-4051 Basel,Switzerland. (Formerly Max Planck InstiTUTE, LEIPZIG (DE))

E-mail address: francesco.ghiraldin@unibas.ch, Francesco.Ghiraldin@mis.mpg.de 